\title{
Residual Effect of Sodium Glucose Cotransporter 2 Inhibitor, Tofogliflozin, on Body Weight After Washout in Japanese Men With Type 2 Diabetes
}

\author{
Hirokazu Kakuda ${ }^{\text {a }}$ Junji Kobayashi ${ }^{\text {b, e }}$, Masaru Sakuraic, Noboru Takekoshid ${ }^{\mathrm{d}}$
}

\begin{abstract}
Background: We investigated the potential mechanism underlying body weight reduction by the sodium glucose cotransporter 2 (SGLT2) inhibitor, tofogliflozin, during treatment and after subsequent washout.

Methods: Ten Japanese men with type 2 diabetes (average age: 66.3 years) were orally administered tofogliflozin $(20 \mathrm{mg} /$ day $)$ for 8 weeks followed by a subsequent 8 -week washout period (16 weeks).

Results: Significant reductions were observed in blood glucose, hemoglobin A1c (HbA1c), uric acid, body weight and waist circumference with an increase in high-molecular weight (HMW) adiponectin at 8 weeks. We also evaluated these markers at 16 weeks and found that unlike HbA1c and uric acid, body weight and HMW adiponectin did not return to baseline levels. To clarify the potential mechanism underlying the body weight reduction during treatment with tofogliflozin ( 8 weeks) and after washout (at 16 weeks), we investigated the correlations between changes from baseline ( 0 week) in body weight and those in waist circumference (or HMW adiponectin). The changes in body weight between 0 weeks versus 8 weeks were not significantly correlated with those in waist circumference or HMW adiponectin. In contrast, changes in body weight between 0 and 16 weeks did show a significant correlation to those in waist circumference and HMW adiponectin.
\end{abstract}

Conclusion: The body weight reduction caused by tofogliflozin may be due to several factors as well as fat reduction at 8 weeks, but is most likely due to fat reduction alone after a subsequent 8 weeks of washout of this agent.

Keywords: Type 2 diabetes; SGLT 2 inhibitor; High-molecular

\footnotetext{
Manuscript submitted October 8, 2018, accepted October 29, 2018

${ }^{a}$ Kakuda Clinic, Takamatsu Na15-1, Kahoku, Ishikawa 929-1215, Japan bDepartment of General Medicine, Kanazawa Medical University, Ishikawa, Japan

'Department of Hygiene, Kanazawa Medical University, Ishikawa, Japan

${ }^{\mathrm{d}}$ Kanazawa Medical University, Ishikawa, Japan

${ }^{e}$ Corresponding Author: Junji Kobayashi, Department of General Medicine, Kanazawa Medical University, 1-1 Daigaku Uchinada, Kahoku, Ishikawa Prefecture 920-0293, Japan. Email: mary@kanazawa-med.ac.jp
}

doi: https://doi.org/10.14740/jocmr3650 weight adiponectin

\section{Introduction}

Sodium/glucose cotransporter-2 (SGLT2) inhibitors are new class of antidiabetic agents for the treatment of type 2 diabetes [1-4]. The SGLT2 inhibitor, tofogliflozin, has 2,900-fold greater selectivity for SGLT2 than SGLT1, and shows the highest selectivity of all clinically developed inhibitors [5]. Similar to other SGLT2 inhibitors [6], treatment with tofogliflozin was shown to be associated with a significant reduction in body weight in addition to a hypoglycemic effect [1,7-11]. To our knowledge, however, there have been no reports on its effect on body weight after withdrawal of this agent. It has been demonstrated that circulating adiponectin levels are lower in obese subjects with high body mass index (BMI), large subcutaneous fat area (SFA) $[12,13]$ and visceral fat area (VFA) [1214]. Several studies have shown that high-molecular weight (HMW) adiponectin was a better predictor than total adiponectin for predicting metabolic disease [15-17]. Studies, including our own, have shown that waist circumference is highly associated with both subcutaneous and visceral fat as measured by computed tomography $(\mathrm{CT})[18,19]$.

Here, we investigated how this agent affects body weight and body composition during treatment and after washout of this agent by measuring HMW adiponectin and waist circumference as well as other metabolic parameters.

\section{Materials and Methods}

This study represents a sub-analysis of the test meal study that we reported previously [1], and the detailed methods were described in that previous report. We briefly describe sample collection. This 16-week, single-arm, pilot clinical study focused on the potential mechanism by which body weight changes occurred after a 8-week oral administration of tofogliflozin (20 $\mathrm{mg}$ /day) and a subsequent 8-week washout of the agent in 10 Japanese men with type 2 diabetic patients who provided written informed consent. On the morning of the day of examination, study subjects did urination completely and took 100 $\mathrm{mL}$ of water at $8 \mathrm{a} . \mathrm{m}$. and then did the second urination right 
Table 1. Patient Background

\begin{tabular}{ll}
\hline Number of subjects & 10 \\
Age (years) & $60.9 \pm 2.8$ \\
HbA1c (\%) & $6.8 \pm 0.2$ \\
Body weight $(\mathrm{kg})$ & $75.4 \pm 4.1$ \\
BMI $\left(\mathrm{kg} / \mathrm{m}^{2}\right)$ & $26.7 \pm 1.1$ \\
Waist circumference $(\mathrm{cm})$ & $91.7 \pm 3.0$ \\
Fasting blood glucose $(\mathrm{mg} / \mathrm{dL})$ & $140.8 \pm 16.2$ \\
Estimated GFR $\left(\mathrm{mL} / \mathrm{min} / 1.73 \mathrm{~m}^{2}\right)$ & $77.3 \pm 4.3$ \\
Systolic blood pressure $(\mathrm{mm} \mathrm{Hg})$ & $139.9 \pm 4.5$ \\
Diastolic blood pressure $(\mathrm{mm} \mathrm{Hg})$ & $83.4 \pm 1.9$ \\
Presence/absence of antihypertensive agent & $5 / 5$ \\
Presence/absence of use of hyperlipemia treatment agent & $5 / 5$ \\
Presence/absence of use of hyperuricemia treatment agent & $1 / 9$ \\
\hline
\end{tabular}

Data are presented as mean \pm SEM.

before taking blood sampling at 0 min followed by the oral ingestion of one pack of the cookie consisting of $75 \mathrm{~g}$ carbohydrate, $28.5 \mathrm{~g}$ fat and $8 \mathrm{~g}$ protein for a total of $592 \mathrm{kcal}$. Subjects spent 10 min taking the cookie with $120 \mathrm{~mL}$ of water and took blood and urine sampling at 60 and $120 \mathrm{~min}$. Tofogliflozin administration started from the next day for 8 weeks and then discontinued followed by another 8 weeks of observation without tofogliflozin.

The effectiveness and safety of this drug were investigated. The same test was conducted 8 weeks after withdrawal, and factors correlated with changes in body weight after administration and after withdrawal were examined.

Selection criteria included age of 20 - 75 years and hemoglobin A1c $(\mathrm{HbA} 1 \mathrm{c})<9.0 \%$.

The exclusion criteria were treatment with SGLT 2 inhibitor within the past 2 months, altered therapy on lipemia and hyperuricemia, other diabetes medications, severe renal dysfunction (estimated glomerular filtration rate (eGFR) $<30 \mathrm{~mL} /$ $\min / 1.73 \mathrm{~m}^{2}$ ), diuretics and a history of cerebral infarction. This work was conducted in accordance with Declaration of Helsinki. Informed consent was obtained from all of the participants.

The institutional review board of Kanazawa Medical University Hospital approved the experimental protocol. This clinical trial was registered with UMIN (registration number: UMIN000015778).

All laboratory tests were conducted by SRL Corporation, Japan. Measurement of the HMW adiponectin was conducted by chemiluminescent enzyme assay (Fujirebio Co., Ltd).

We investigate the correlations between changes in body weight between 0 weeks versus 8 weeks to those in waist circumference or HMW adiponectin. We performed the same analysis between 0 weeks versus 16 weeks. Statistical analyses were performed to determine the significance of changes before and after administration of each measurement item and the amounts of change before administration and after withdrawal. Parameters with a normal distribution are shown as the mean \pm standard error and were analyzed by the corresponding $t$-test. Items with a non-normal distribution are shown as the median value $(25 \%, 75 \%)$ and were analyzed using Wilcoxon's signed-rank test. Bivariate correlations were determined using Pearson's product moment correlation coefficient. In all analyses, $P<0.05$ was taken to indicate statistical significance. This study was conducted based on a doctor-initiated clinical research contract with Kowa Co., Ltd.

\section{Results}

The patient background characteristics are shown in Table 1. The changes in parameters before and after administration of tofogliflozin and after washout are shown in Table 2. HbA1c decreased significantly from $6.8 \%$ before administration to $6.5 \%$ after administration of tofogliflozin. Body weight, waist circumference, HMW adiponectin and serum uric acid levels also improved significantly after administration of tofogliflozin. Significant increases were observed in hematocrit and red blood cell (RBC) after administration, and significant increases were maintained even after withdrawal. The area under the curve (AUC) of urinary glucose after test meal loading showed a significant increase after administration, but urinary volume AUC only tended to increase. Significant differences in all of the markers disappeared after withdrawal of the drug.

Next, we investigated factors potentially affecting body weight changes after administration or withdraw of tofogliflozin. No significant correlations between body weight change and waist circumference change were observed after 8 weeks of administration, but a strong positive correlation was observed after 8 weeks of withdrawal (Fig. 1). Similarly, no significant correlation was observed between body weight change and HMW adiponectin change after 8 weeks of administration, but a significant inverse correlation was observed after 8 weeks of washout (Fig. 2). We examined the correlations between body weight and hematocrit changes (Fig. 3) and those between body weight and 2-hour urine volume changes (Fig. 
Table 2. Changes in Each Parameter Versus Baseline

\begin{tabular}{|c|c|c|c|c|c|}
\hline & \multirow{2}{*}{$\begin{array}{l}\text { Baseline } \\
\text { 0 week }\end{array}$} & \multicolumn{2}{|c|}{ After treatment } & \multicolumn{2}{|c|}{ After washout } \\
\hline & & 8 weeks & Changes from baseline & 16 weeks & Changes from baseline \\
\hline $\mathrm{HbAlc}(\%)$ & $6.8 \pm 0.2$ & $6.5 \pm 0.2$ & $-0.3 *$ & $6.9 \pm 0.3$ & 0.05 \\
\hline Body weight (kg) & $75.4 \pm 4.1$ & $73.0 \pm 4.2$ & $-2.4 * * *$ & $74.0 \pm 4.7$ & -1.4 \\
\hline Waist circumference $(\mathrm{cm})$ & $91.7 \pm 3.0$ & $89.3 \pm 3.0$ & $-2.4 * *$ & $90.3 \pm 3.8$ & -1.4 \\
\hline Ht $(\%)$ & $43.92 \pm 1.1$ & $46.84 \pm 0.83$ & $2.92 * * *$ & $45.39 \pm 1.0$ & $1.47 * * *$ \\
\hline $\mathrm{RBC}(/ \mu \mathrm{L})$ & $480.4 \pm 13.8$ & $510.3 \pm 10.28$ & $29.9 * * *$ & $492.2 \pm 11.3$ & $11.8^{*}$ \\
\hline \#Urine glucose AUC120 min (g) & $3.8 \pm 2.5$ & $24.7 \pm 4.8$ & $20.9 * *$ & $1.7 \pm 1$ & -2.1 \\
\hline${ }^{\#}$ Urine volume AUC120 min (mL) & $224.7 \pm 50.2$ & $400.4 \pm 73.4$ & 175.7 & $275.1 \pm 98.1$ & 50.4 \\
\hline
\end{tabular}

Data are expressed as mean \pm SEM or median (1st, 3rd quartile). ${ }^{*} \mathrm{P}<0.05,{ }^{* *} \mathrm{P}<0.01,{ }^{* *} \mathrm{P}<0.001$ (versus baseline) with paired $t$-test or Wilcoxon's signed-rank tests. \#Urine was collected during $120 \mathrm{~min}$ after loading test meal and volume and glucose content were measured [1].

4) in the same manner. However, no significant correlation was observed either after 8 weeks of administration or after 8 weeks of washout.

In addition to the analysis regarding 0 - 8 weeks and 0 - 16 weeks, we also conducted same analysis on the correlation of body weight change to waist circumference change (or HMW adiponectin change) during 8 - 16 weeks. There were positive correlations between body weight change and waist circumference change $(r=0.82, P=0.004)$, while no correlations were observed between body weight change and HMW adiponectin change $(\mathrm{r}=-0.22, \mathrm{P}=0.55)$.

There were no side effects during the study period.

\section{Discussion}

The main findings of this study were as follows: 1) significant correlations between body weight change and waist circumfer- ence change were observed after 8 weeks of washout, which were not seen during treatment with tofogliflozin; 2) significant correlations between body weight change and HMW adiponectin changes were observed after 8 weeks of washout, which were not seen during treatment with tofogliflozin.

SGLT2 inhibitors are novel medications that lower blood glucose by promoting urinary glucose excretion and improving metabolism, showing a number of effects such as weight reduction, lowering of blood pressure, uric acid reduction, etc., in addition to hypoglycemic effects [1-4]. Initially, it was suggested that calorie loss of about $320 \mathrm{kcal}$ caused by $80 \mathrm{~g}$ of urinary glucose per day may be solely responsible for the body weight loss by SGLT2 inhibitors [20]. Other mechanisms for body weight loss were also considered because weight reductions were also observed even when administered to renal dysfunction patients with impaired urine secretion [21]. One suggested mechanism was the effect of decreasing body fluid volume due to the osmotic diuretic ef-
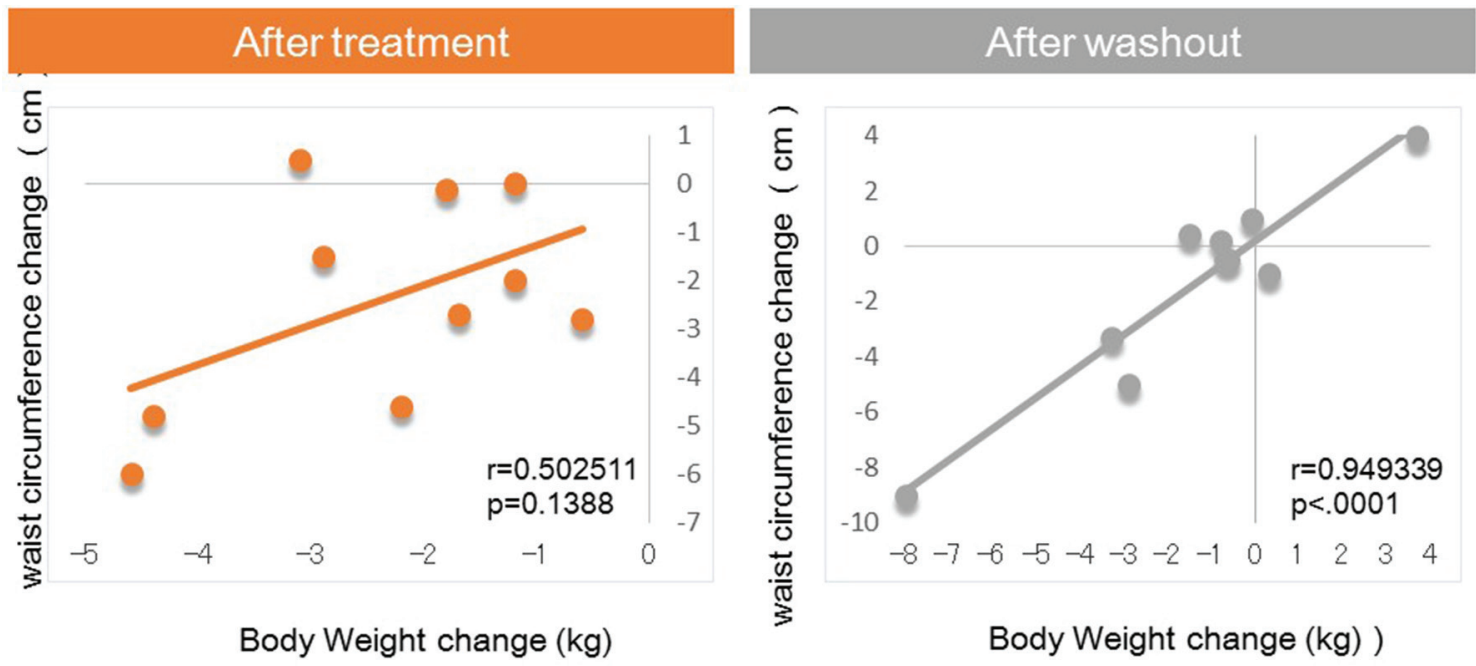

Figure 1. Correlation between body weight changes and waist circumference changes from baseline. 


\section{After treatment}

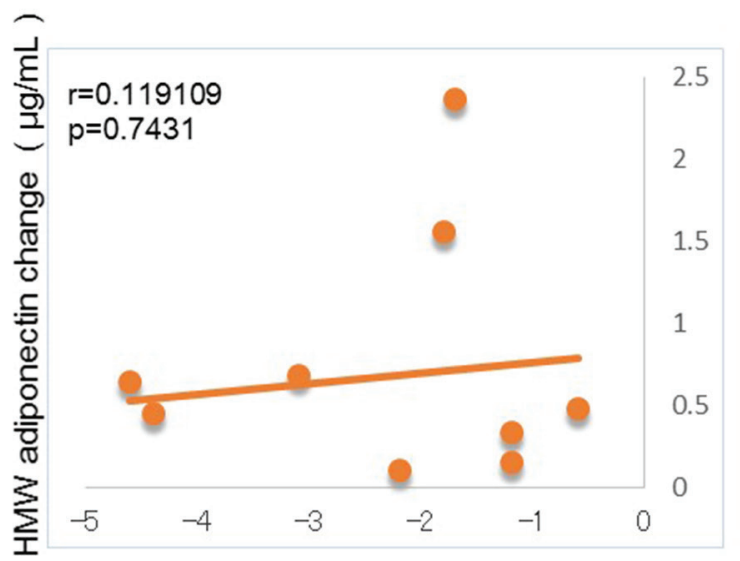

Body Weight change $(\mathrm{kg})$

\section{After washout}

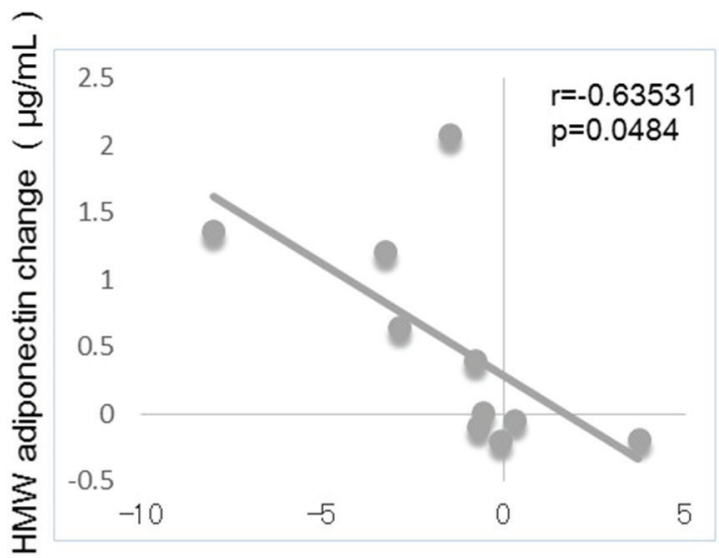

Body Weight change $(\mathrm{kg})$

Figure 2. Correlation between body weight changes and HMW adiponectin changes from baseline.

fect as a result of accelerated urinary glucose excretion, while another mechanism was suggested to be the promotion of fat decomposition $[22,23]$.

In the present study, after 8 weeks of administration, there were no correlations of body weight change with waist circumference or HMW adiponectin change. In contrast, after 8 weeks of washout, there were significant correlations between body weight change and changes in waist circumference and HMW adiponectin. These results suggested that various factors other than fat loss, most likely body fluid volume reduction, may contribute to body weight reduction after 8 weeks of tofogliflozin administration, while after 8 weeks of washout, fat loss may be the sole factor contributing to weight loss given the previous studies on the relations of body fat accumulation to adiponectin [12-17] (Fig. 5). Our current finding that during treatment with tofogliflozin there were no correlations of body weight change with HMW adiponectin change is not inconsistent with the recent report by Garvey et al [24] showing that the increase in adiponectin with another SGLT2 inhibitor, canagliflozin, was independent of changes in body weight. Thus, we presume that body weight reductions during treatment with SGLT2 inhibitor are combination of body fluid volume reduction and body fat reduction.

There were no correlations of body weight change to hematocrit or urine volume after either 8 weeks of administration or after 8 weeks of washout.

The limitation of this study is that the sample size was small, there was no control group and that body composition was not directly measured. Further study with larger sample size and with control group is needed to confirm the findings in our present pilot study.

In conclusion, in addition to glycemic control, the SGLT2

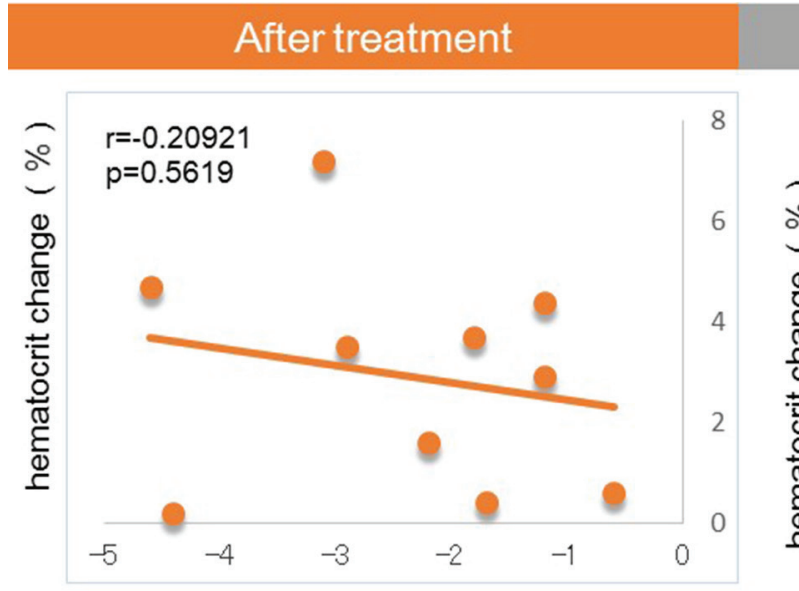

Body Weight change $(\mathrm{kg})$
After washout

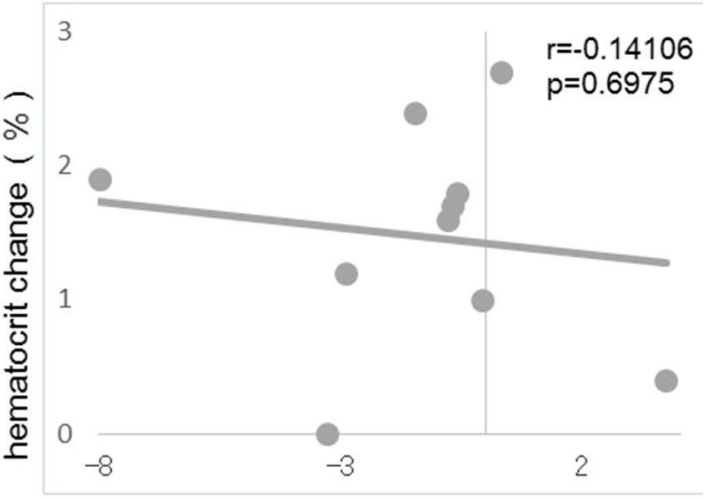

Body Weight change $(\mathrm{kg})$

Figure 3. Correlation between body weight changes and hematocrit changes from baseline. 

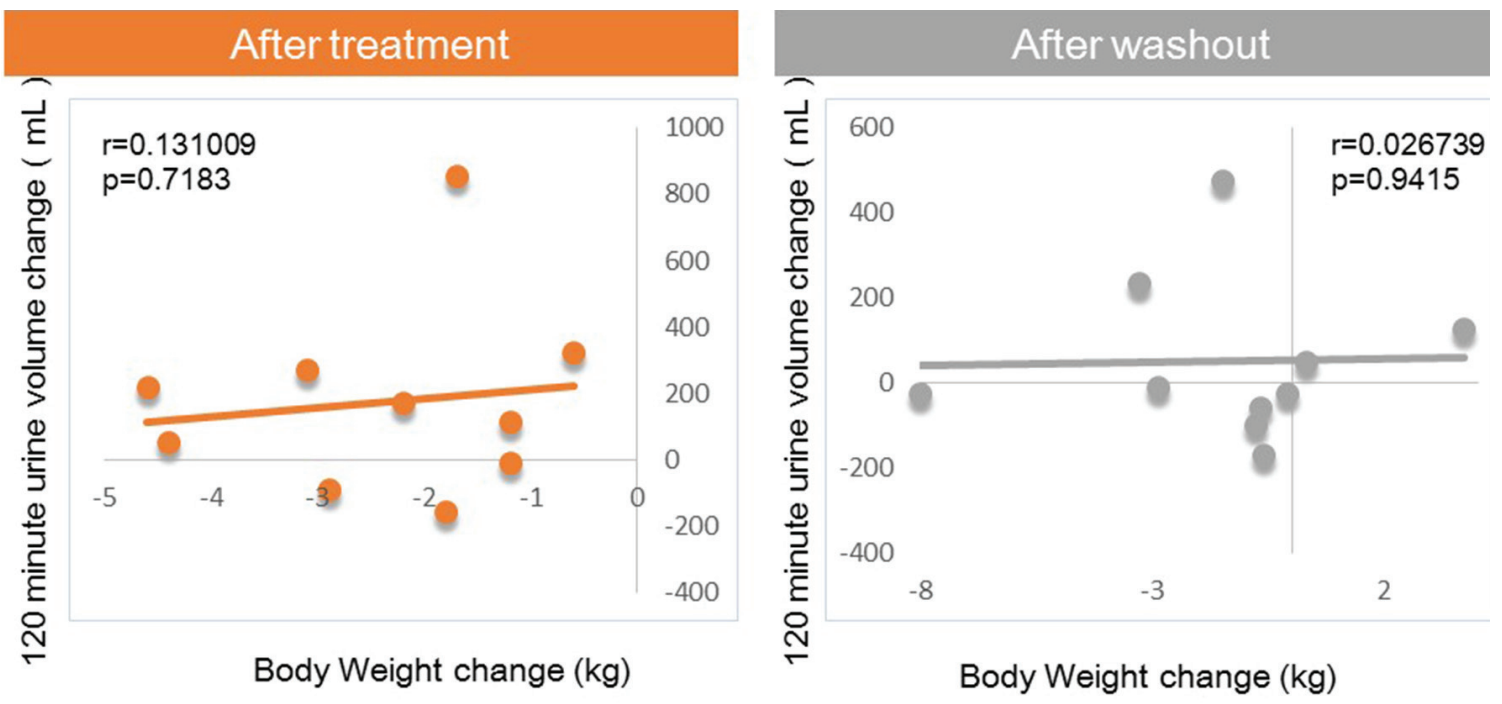

Figure 4. Correlation between body weight changes and 120-min urine volume changes from baseline.

inhibitor, tofogliflozin, was confirmed to be useful for decreasing body weight, and this body weight loss effect remained even after discontinuation of the drug. The body weight reduc- tion by tofogliflozin may be due to several factors as well as fat reduction at 8 weeks, but the reduction in body weight is most likely due to fat reduction alone after 8 weeks of washout.

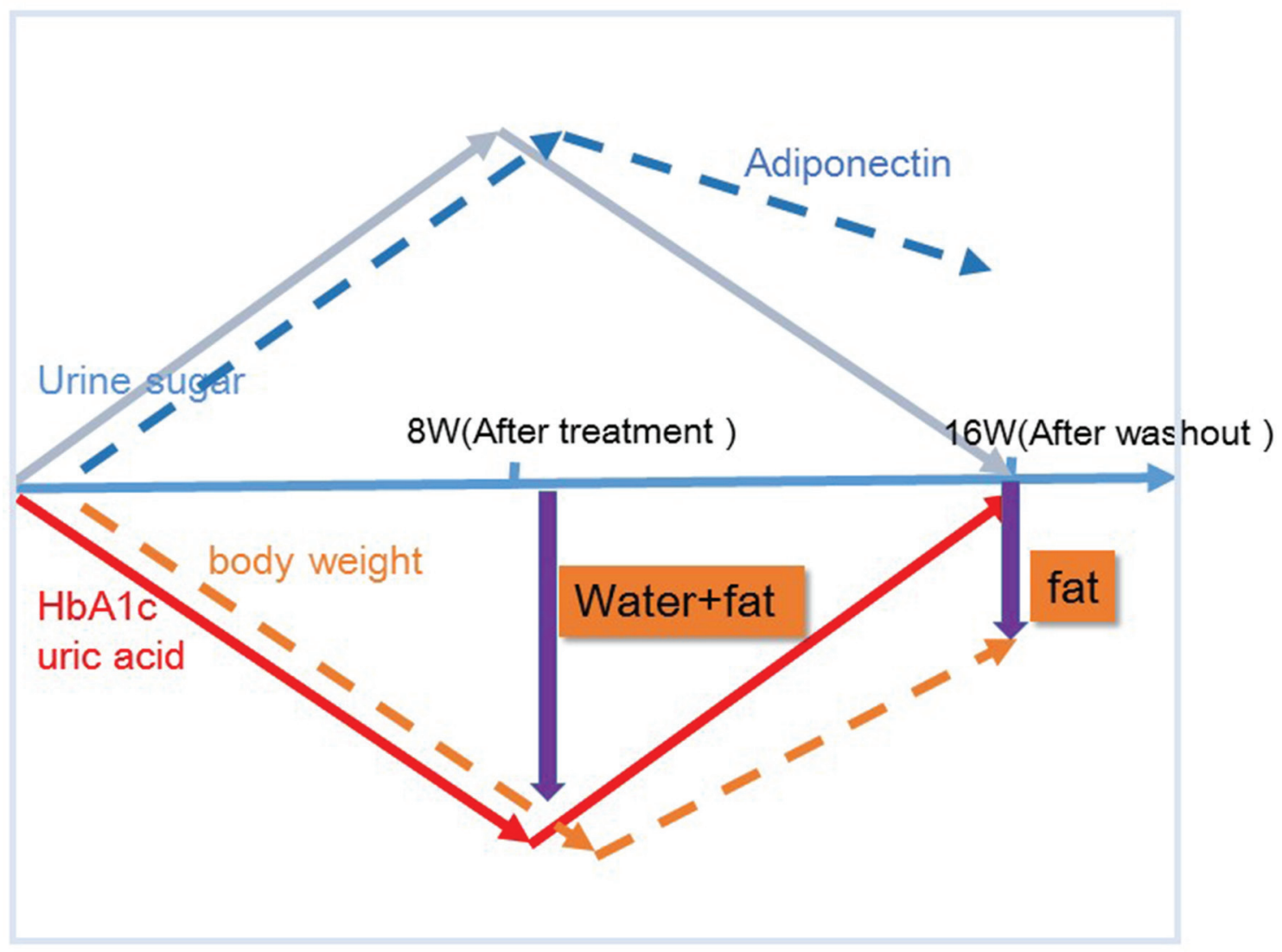

Figure 5. Proposed chart for changes in body weight and potential body composition after treatment and after washout of tofogliflozin. The mechanism by which body weight reductions caused by tofogliflozin may be due to several factors as well as fat reductions at 8 weeks, but those may be due most likely to fat reductions alone after a subsequent 8 weeks of washout (16 week) of this agent. 


\section{Conflict of Interest}

We have received research funding from Kowa Co., Ltd.

\section{References}

1. Kakuda H, Kobayashi J, Sakurai M, Kakuda M, Takekoshi N. The effect of tofogliflozin treatment on postprandial glucose and lipid metabolism in Japanese men with type 2 diabetes: A Pilot Study. J Clin Med Res. 2017;9(5):403409.

2. Nair S, Wilding JP. Sodium glucose cotransporter 2 inhibitors as a new treatment for diabetes mellitus. J Clin Endocrinol Metab. 2010;95(1):34-42.

3. Vasilakou D, Karagiannis T, Athanasiadou E, Mainou M, Liakos A, Bekiari E, Sarigianni M, et al. Sodiumglucose cotransporter 2 inhibitors for type 2 diabetes: a systematic review and meta-analysis. Ann Intern Med. 2013;159(4):262-274.

4. Misra M. SGLT2 inhibitors: a promising new therapeutic option for treatment of type 2 diabetes mellitus. J Pharm Pharmacol. 2013;65(3):317-327.

5. Suzuki M, Honda K, Fukazawa M, Ozawa K, Hagita H, Kawai T, Takeda M, et al. Tofogliflozin, a potent and highly specific sodium/glucose cotransporter 2 inhibitor, improves glycemic control in diabetic rats and mice. J Pharmacol Exp Ther. 2012;341(3):692-701.

6. Barnett AH. Impact of sodium glucose cotransporter 2 inhibitors on weight in patients with type 2 diabetes mellitus. Postgrad Med. 2013;125(5):92-100.

7. Ikeda S, Takano Y, Cynshi O, Tanaka R, Christ AD, Boerlin V, Beyer U, et al. A novel and selective sodiumglucose cotransporter-2 inhibitor, tofogliflozin, improves glycaemic control and lowers body weight in patients with type 2 diabetes mellitus. Diabetes Obes Metab. 2015;17(10):984-993.

8. Kamei S, Iwamoto M, Kameyama M, Shimoda M, Kinoshita T, Obata A, Kimura T, et al. Effect of tofogliflozin on body composition and glycemic control in Japanese subjects with type 2 diabetes mellitus. J Diabetes Res. 2018;2018:6470137.

9. Terauchi Y, Tamura M, Senda M, Gunji R, Kaku K. Longterm safety and efficacy of tofogliflozin as add-on to insulin in patients with type 2 diabetes: Results from a 52week, multicentre, randomized, double-blind, open-label extension, Phase 4 study in Japan (J-STEP/INS). Diabetes Obes Metab. 2018;20(5):1176-1185.

10. Hirose S, Nakajima S, Iwahashi Y, Seo A, Takahashi T, Tamori Y. Impact of the 8-week administration of tofogliflozin for glycemic control and body composition in Japanese patients with type 2 diabetes mellitus. Intern Med. 2016;55(22):3239-3245.

11. Kaku K, Watada H, Iwamoto Y, Utsunomiya K, Terauchi Y, Tobe K, Tanizawa Y, et al. Efficacy and safety of monotherapy with the novel sodium/glucose cotransporter-2 inhibitor tofogliflozin in Japanese patients with type 2 diabetes mellitus: a combined Phase 2 and 3 randomized, placebo-controlled, double-blind, parallel-group comparative study. Cardiovasc Diabetol. 2014;13:65.

12. Arita Y, Kihara S, Ouchi N, Takahashi M, Maeda K, Miyagawa J, Hotta K, et al. Paradoxical decrease of an adipose-specific protein, adiponectin, in obesity. Biochem Biophys Res Commun. 1999;257(1):79-83.

13. Ryo M, Nakamura T, Kihara S, Kumada M, Shibazaki S, Takahashi M, Nagai M, et al. Adiponectin as a biomarker of the metabolic syndrome. Circ J. 2004;68(11):975-981.

14. Okauchi Y, Kishida K, Funahashi T, Noguchi M, Ogawa T, Ryo M, Okita K, et al. Changes in serum adiponectin concentrations correlate with changes in BMI, waist circumference, and estimated visceral fat area in middle-aged general population. Diabetes Care. 2009;32(10):e122.

15. Pajvani UB, Hawkins M, Combs TP, Rajala MW, Doebber T, Berger JP, Wagner JA, et al. Complex distribution, not absolute amount of adiponectin, correlates with thiazolidinedione-mediated improvement in insulin sensitivity. J Biol Chem. 2004;279(13):12152-12162.

16. Hara K, Horikoshi M, Yamauchi T, Yago H, Miyazaki $\mathrm{O}$, Ebinuma H, Imai Y, et al. Measurement of the highmolecular weight form of adiponectin in plasma is useful for the prediction of insulin resistance and metabolic syndrome. Diabetes Care. 2006;29(6):1357-1362.

17. Aso Y, Yamamoto R, Wakabayashi S, Uchida T, Takayanagi K, Takebayashi K, Okuno T, et al. Comparison of serum high-molecular weight (HMW) adiponectin with total adiponectin concentrations in type 2 diabetic patients with coronary artery disease using a novel enzymelinked immunosorbent assay to detect HMW adiponectin. Diabetes. 2006;55(7):1954-1960.

18. Oka R, Miura K, Sakurai M, Nakamura K, Yagi K, Miyamoto $\mathrm{S}$, Moriuchi T, et al. Impacts of visceral adipose tissue and subcutaneous adipose tissue on metabolic risk factors in middle-aged Japanese. Obesity (Silver Spring). 2010;18(1):153-160.

19. Kim JH, Doo SW, Cho KS, Yang WJ, Song YS, Hwang J, Hong SS, et al. Which anthropometric measurements including visceral fat, subcutaneous fat, body mass index, and waist circumference could predict the urinary stone composition most? BMC Urol. 2015;15:17.

20. Nishimura R, Osonoi T, Kanada S, Jinnouchi H, Sugio $\mathrm{K}$, Omiya H, Ubukata M, et al. Effects of luseogliflozin, a sodium-glucose co-transporter 2 inhibitor, on 24-h glucose variability assessed by continuous glucose monitoring in Japanese patients with type 2 diabetes mellitus: a randomized, double-blind, placebo-controlled, crossover study. Diabetes Obes Metab. 2015;17(8):800-804.

21. Kohan DE, Fioretto P, Tang W, List JF. Long-term study of patients with type 2 diabetes and moderate renal impairment shows that dapagliflozin reduces weight and blood pressure but does not improve glycemic control. Kidney Int. 2014;85(4):962-971.

22. Iizuka T, Iemitsu K, Takihata M, Takai M, Nakajima S, Minami N, Umezawa S, et al. Efficacy and safety of ipragliflozin in Japanese patients with type 2 diabetes: interim outcome of the ASSIGN-K study. J Clin Med Res. 2016;8(2):116-125.

23. Obata A, Kubota N, Kubota T, Iwamoto M, Sato H, 
Sakurai Y, Takamoto I, et al. Tofogliflozin improves insulin resistance in skeletal muscle and accelerates lipolysis in adipose tissue in male mice. Endocrinology. 2016;157(3):1029-1042.
24. Garvey WT, Van Gaal L, Leiter LA, Vijapurkar U, List J, Cuddihy R, Ren J, et al. Effects of canagliflozin versus glimepiride on adipokines and inflammatory biomarkers in type 2 diabetes. Metabolism. 2018;85:32-37. 